\title{
Focal gamma activity in frontal control regions revealed using intraoperative electrocorticography
}

Moataz Assem ${ }^{1 *}$, Michael G. Hart ${ }^{2}$, Pedro Coelho ${ }^{3}$, Rafael Romero-Garcia ${ }^{4}$, Alexa McDonald $^{5}$, Emma Woodberry ${ }^{5}$, Robert C. Morris ${ }^{2}$, Stephen J. Price ${ }^{6}$, John Suckling ${ }^{4,7,8}$, Thomas Santarius ${ }^{2,9}$, John Duncan ${ }^{1,10}$, Yaara Erez ${ }^{1}$

1 Medical Research Council, Cognition and Brain Sciences Unit, University of Cambridge

2 Department of Neurosurgery, Cambridge University Hospitals NHS Foundation Trust

3 Neurophys Limited

4 Department of Psychiatry, University of Cambridge

5 Department of Neuropsychology, Cambridge University Hospitals NHS Foundation Trust

6 Division of Neurosurgery, Department of Clinical Neurosciences, University of Cambridge.

7 Behavioural and Clinical Neuroscience Institute, University of Cambridge

8 Cambridge and Peterborough NHS Foundation Trust

9 Department of Physiology, Development and Neuroscience, University of Cambridge

10 Department of Experimental Psychology, University of Oxford

*corresponding author

Moataz Assem

Moataz.assem@mrc-cbu.cam.ac.uk

15 Chaucer road, Cambridge, UK, CB2 7EF 


\section{Abstract}

How the frontal cortex is anatomically and functionally organized to control cognition remains puzzling. Numerous non-invasive brain imaging studies relate cognitive control to a localized set of frontal regions, part of a wider fronto-parietal network (FPN), that show increases in functional MRI (fMRI) signal during the performance of multiple cognitively demanding tasks. Lesions implicating frontal control regions lead to disorganized behaviour. However, the fMRI BOLD signal is an indirect measure of neuronal activity and represents evidence from a single modality. This has led to limited clinical translation of fMRI findings e.g. to guide the surgical resection of brain tumours. Here we sought supporting evidence for lateral frontal control regions using electrocorticography (ECoG). We recorded electrophysiological activity from electrodes placed on the lateral frontal cortex in patients undergoing awake craniotomy for glioma resection. During surgery, patients performed two verbal executive-related counting tasks with a difficulty level manipulation, closely adapting difficulty manipulations in fMRI studies of cognitive control. We performed spectral analysis focusing on the gamma range $(30-250 \mathrm{~Hz})$ due to mounting evidence of its value as an index of local cortical processing. Comparing hard versus easy demands revealed circumscribed frontal regions with power increases in the gamma range. This contrasted with spatially distributed power decreases in the beta range $(12-30 \mathrm{~Hz})$. Further, electrodes showing significant gamma power increases were more likely to occur within a canonical fMRI-defined FPN and showed stronger gamma power increases compared to electrodes outside the FPN, even at the single patient level. Reinforcing the need for careful task manipulation, an easy versus baseline comparison, which includes factors such as speech output, produced gamma changes over a wider area. Thus, using similar task difficulty manipulations, ECoG and fMRI signals converged on delineating lateral frontal control regions. These findings open the door for extending clinical functional mapping to the domain of cognitive control during awake neurosurgery. 


\section{Introduction}

Essential to goal-directed behaviour is our ability to regulate and control cognitive processes, allocating resources appropriately to successfully achieve a goal. Cognitive control is an umbrella term for processes such as selective attention, working memory, set shifting, response inhibition, conflict monitoring, problem solving, and goal-directed behaviour (Diamond, 2013). In the human brain, a specific network of cortical areas, largely fronto-parietal, is hypothesised to support such control processes across multiple cognitive domains (Duncan and Owen, 2000; Cole and Schneider, 2007; Duncan, 2010). Damage to fronto-parietal control regions is associated with disorganized behaviour and poor fluid intelligence scores (Glascher et al., 2010, 2012, Woolgar et al., 2010, 2018; Warren et al., 2014). Ample evidence from functional magnetic resonance imaging (fMRI) studies associate increased BOLD co-activation of fronto-parietal areas with increased cognitive demands across multiple tasks including attention, working memory, task switching and task-relevant information selection (Corbetta and Shulman, 2002; Fedorenko et al., 2013; Muhle-Karbe et al., 2014; Erez and Duncan, 2015; Cole et al., 2016; Jackson et al., 2016; Shashidhara et al., 2019, 2020; Assem et al., 2020). Furthermore, fronto-parietal regions form an integrated network, as reflected in correlation of their timeseries during resting-state fMRI, forming the canonical fronto-parietal network (FPN) (Power et al., 2011; Yeo et al., 2011; Assem et al., 2020).

Much uncertainty remains, however, over precise localisation of cognitive control function within the human frontal lobe using fMRI on its own. Because the neuronal origins of fMRI BOLD signals are not well understood, and the reliability of its findings are sensitive to the use of non-optimal analysis methods (Coalson et al., 2018; Botvinik-Nezer et al., 2020), its findings are yet to be translated into clinical practice. Here we sought supporting evidence for specific cognitive control regions in the lateral frontal cortex from a different, clinically-relevant modality, electrocorticography (ECoG), recorded during awake tumour surgery. During surgery, functional mapping is used to inform clinical decisions, guide resection and preserve cognitive function following surgery. However, functional mapping is mostly used for motor function and language (Crone et al., 2006). Identifying the 
medRxiv preprint doi: https://doi.org/10.1101/2021.08.13.21261980; this version posted August 13, 2021. The copyright holder for this preprint

regions that support cognitive control intraoperatively could provide valuable information to guide resection and prevent commonly-seen deficits following surgery (van Loon et al., 2015). In ECoG data recorded during surgery, we sought evidence for specific FPN activity linked to cognitive control demand.

Compared to electroencephalogram (EEG) and magnetoencephalogram (MEG), ECoG offers both much-improved spatial resolution and the ability to detect local field potential (LFP) modulations within the broadband gamma frequency range $(30-250 \mathrm{~Hz})$. Accumulating evidence has emphasized the role of broadband gamma activity as an index for local cortical processing across a wide range of functional domains, but activity in this frequency range cannot usually be detected noninvasively because of its low amplitude and the low-pass filter induced by the scalp (Crone et al., 2006; Lachaux et al., 2012). In the cognitive control domain, evidence for the role of broadband gamma activity comes from invasive studies in both humans and animals. Increases in broadband gamma have long been known to reflect a heightened attentive state (Bouyer et al., 1981) and have been linked to processing of attended stimuli in both early sensory and fronto-parietal regions (Fries, 2001; Ray et al., 2008; Szczepanski et al., 2014; Helfrich and Knight, 2016). Increases in gamma power were also associated with working memory load, and during delay periods (Howard, 2003; Mainy et al., 2007), and sustained gamma responses were observed in frontal regions (Haller et al., 2018). Responses to more abstract rules have been associated with frontal gamma increases (Voytek et al., 2015). A common limitation for the above-mentioned studies is their lack of anatomical specificity when localizing gamma activity, mostly allocating it to broad labels such as "dorsolateral prefrontal cortex". Such association cortices are rich in functionally heterogeneous regions (Glasser et al., 2016), making it difficult to relate electrophysiological and functional neuroimaging findings.

Increases in power in the gamma range are often accompanied by decreases in power in lower frequencies, in particular in the beta range $(12-30 \mathrm{~Hz})$. This has been demonstrated using different tasks and across many cortical regions including motor, sensory, visual and auditory tasks (Crone et al., 2006; Hermes et al., 2012b; Podvalny et al., 2015). For example, in motor and sensory cortex, hand movements were associated with both increases in high-frequency band power as well as decreases in the beta range (Hermes et al., 2012b). More recently, increases in the gamma range and decreases in the beta range in the prefrontal cortex of non-human 
primates have been associated with working memory, a central component of cognitive control processes (Lundqvist et al., 2016).

To identify an electrophysiological signature of domain-general control regions in the human brain, we used ECoG during awake brain surgeries while patients performed two tasks with a varying level of control demand. Following a previous demonstration of the feasibility of this approach (Erez et al., 2021), here we use dense coverage of the frontal cortex to characterize the power modulations associated with increased cognitive demand, and directly link them to the FPN. Previous studies demonstrated correlates between the fMRI BOLD response and broadband gamma power (Logothetis et al., 2001; Mukamel et al., 2005; Siero et al., 2013). Taken together with reports of power changes across a variety of tasks related to cognitive control processes (Crone et al., 2006; Lachaux et al., 2012), we predicted that increased control demand will be associated with power increases in the broadband gamma range, especially within the FPN, accompanied by power decreases in the beta range. 


\section{Methods}

\section{Patient recruitment}

Twenty-one patients were recruited from the pool of glioma patients who have been indicated to undergo awake resections of their tumours at the Department of Neurosurgery at [redacted to comply with preprint server policy]. Data from thirteen patients were complete and were included in the study (age range 22-56; 6 males; see Table 1 for patient demographics). Data from the remaining eight patients were excluded either due to technical difficulties $(n=6)$ or an inability to perform the tasks during the surgery $(n=2)$. All study procedures were approved by the East of England - Cambridge Central Research Ethics Committee (REC reference 16/EE/0151). All patients gave written informed consent to participate and were aware that the research would not benefit themselves, or impact their clinical care before, during or after surgery.

\section{Experimental procedures}

The patients were familiarized with the tasks during standard pre-operative clinic visits and as part of a pre-operative research-dedicated assessment. During the surgery and following the craniotomy, testing was performed after the patient had been awakened and prior to tumour resection. In one patient, the testing was performed after partial resection due to clinical considerations. Figures 1a,b illustrate the intraoperative setup and cognitive tasks. During testing, all personnel in the surgical theatre were asked to limit their conversations to minimize disruptions. Patients performed one baseline task and two cognitive tasks. For the baseline task, the patients were asked to stay calm and remain silent for a period of 2-3 minutes (rest). The two cognitive tasks were simple counting ( 1 to 20 ; easy) and alternate counting and reciting the alphabet (1, a, 2, b, 3, c, up to 20; hard). Each task condition was repeated for 2-5 trials (median for both $=4$ trials) based on each patient's ability and time constraints during the surgery. Trial onset and offset markers were manually recorded on the acquisition system. Trial durations were $20.1 \pm 7.4 \mathrm{~s}$ and $29.4 \pm 9.4 \mathrm{~s}$ for the easy and hard conditions, respectively. Most 
patients were instructed to alternate between trials of the easy and hard conditions, though on a few occasions some easy/hard trials were performed in succession. Only correctly performed trials (i.e. no errors in simple or alternate counting) were included in the analysis (e.g. a failed hard trial that was excluded: 1, a, 2, b, 3, b, 4, b, $5, \mathrm{~b}, 6, \mathrm{~b} \ldots)$.

\section{MRI acquisition}

MRI data were acquired pre-operatively using a Siemens Magnetom Prisma-fit 3 Tesla MRI scanner and 16-channel receive-only head coil (Siemens AG, Erlangen, Germany). Structural anatomic images were acquired using a T1-weighted (T1w) MPRAGE sequence $(F O V=256 \mathrm{~mm} \times 240 \mathrm{~mm} \times 176 \mathrm{~mm}$; voxel size $=1 \mathrm{~mm}$ isotropic; repetition time $(\mathrm{TR})=2300 \mathrm{~ms}$; echo time $(\mathrm{TE})=2.98 \mathrm{~ms}$; flip angle $=9$ degrees).

\section{Electrode localization}

The extent of craniotomy of all patients was determined by clinical considerations to allow for tumour resection. Based on the craniotomy size and location, one to three electrode strips were placed on the cortical surface in regions judged by the neurosurgeon to be healthy (i.e. macroscopically not containing tumour). Strips placed on the tumour or outside of the frontal and motor cortices were excluded from analysis. Each strip was composed of four electrodes. Two types of strips were used with electrode diameter either $5 \mathrm{~mm}$ (MS04R-IP10X-0JH, Ad-Tech, Medical Instruments corporation, WI, USA) or $3 \mathrm{~mm}$ (CORTAC 2111-04-081, PMT Corporation, MN, USA). For both strip types, electrode spacing was $10 \mathrm{~mm}$ centre to centre.

Electrode locations were determined either using (1) an automated method with a probe linked to a stereotactic neuronavigation system (StealthStation ${ }^{\circledR}$ S7® System, Medtronic, Inc, 24 Louisville, CO, USA) or (2) a semi-manual grid method using intraoperative photographs and a grid-like delineation of cortical sulci and gyri. Most electrodes (51/79) were localized using the automated method, and due to occasional technical limitations, 28 electrodes were localized using the grid method. Both methods are detailed below. 
(1) Stereotactic neuronavigation: A hand-held probe was placed at the centre of each electrode, automatically registering its physical coordinates, using the neuronavigation system, to the patient's native high resolution preoperative $\mathrm{T} 1 \mathrm{w}$ scan. In some cases, coordinate data were available for only two or three out of the four electrodes in each strip. This was due to either time constraints during the surgery or because an electrode was located underneath the skull, excluding probe placement. Each patient's native T1w scan was linearly co-registered with the MNI template volume at $2 \mathrm{~mm}$ resolution using FLIRT as implemented in FSL (Jenkinson et al., 2012) using 12 degrees of freedom (full set affine transformation) and the correlation ratio cost function. The resulting native-to-MNI transformation matrix was then used to convert native electrode coordinates to MNI coordinates.

(2) The grid method: This follows the method described in (Havas et al., 2015) and (Ojemann et al., 1989). (a) Visible major sulci were delineated on the intraoperative photographs: precentral sulcus, sylvian fissure, inferior and superior frontal sulci. Spaces between these sulci were populated by vertical lines $(1.5 \mathrm{~cm}$ apart) to create a grid-like structure. (b) A grid was created in the same way on a template cortical reconstruction of the MNI volumetric map (reconstructed using the HCP structural preprocessing pipeline 4.0.0; https://github.com/WashingtonUniversity/HCPpipelines). (c) MNI coordinates for each electrode were extracted by manually marking its approximate location on the template cortical grid while visualized using the Connectome Workbench v1.4.2 (https://www.humanconnectome.org/software/get-connectome-workbench). As the template cortical reconstruction is co-registered with its MNI volumetric version, it facilitated the automatic transformation of any point marked on the surface back to its MNI volumetric coordinates.

Electrode displacements due to brain shifts following the craniotomy were compensated for by back-projecting the electrode locations onto the cortical surface along the local norm vector (Hermes et al., 2010) as implemented in the fieldtrip (v20160629) protocol for human intracranial data (Stolk et al., 2018).

\section{Electrophysiological data acquisition and analysis}

Data were recorded using a 32-channel amplifier (Medtronic Xomed, Jacksonville, FS, USA) sampled at $10 \mathrm{KHz}$. Potential sources of electrical noise such as the 
medRxiv preprint doi: https://doi.org/10.1101/2021.08.13.21261980; this version posted August 13, 2021. The copyright holder for this preprint

surgical microscope, patient warming blanket, and IV pumps were identified and repositioned to avoid signal contamination. The data were recorded via dedicated channels on the acquisition system and two Butterworth online filters were applied: a high-pass filter at $1 \mathrm{~Hz}$ and a low-pass filter at $1500 \mathrm{~Hz}$. A ground needle electrode was connected to the deltoid muscle and the electrodes were referenced to a mid-frontal (Fz) spiral scalp EEG electrode.

Data were analysed offline using EEGLAB (v13.6.5b) and custom MATLAB scripts. The data were downsampled to $2 \mathrm{kHz}$ then re-referenced using a bipolar scheme to detect any activity changes with the highest spatial resolution as well as to avoid contamination of high frequency signals by scalp muscle artefacts detected by the $\mathrm{Fz}$ electrode. The last electrode on the strip was excluded from analysis; i.e. for a four-electrode strip, electrode pairs 1-2, 2-3 and 3-4 were used and assigned to electrode positions 1, 2 and 3, respectively. The location of electrode 4 was discarded. Thus, out of the original 79 electrodes, re-referenced data from 59 were used for further analysis (Figure 1c). Out of these 59 electrodes, 41 were on the left hemisphere and 18 on the right. 25 electrodes were placed on the middle frontal gyrus (MFG), 28 on the inferior frontal gyrus (IFG) and on 6 on motor cortex.

A notch filter was applied at $50 \mathrm{~Hz}$ and its harmonics to remove line noise. Notch filtering was also applied at $79 \mathrm{~Hz}$ and its harmonics to remove additional noise observed in the data, probably due to equipment in the surgical theatre. Data were then bandpass filtered into 6 classical frequency bands (delta: 1-4 Hz, theta: 4$8 \mathrm{~Hz}$, alpha: 8-12 Hz, beta: 12-30 Hz, low gamma (LG): 30-70 Hz, high gamma (HG): 70-250 Hz). Instantaneous power of the timeseries was obtained by squaring the absolute amplitude envelope of the Hilbert transformed data.

The power timeseries data were then segmented into separate conditions and trials. Because trial onset and offset markers were manually recorded, $2 \mathrm{~s}$ from the beginning and end of the rest trial and $1 \mathrm{~s}$ from each task trial were excluded to account for human reaction time related error. For the hard trials (alternate numbers/letters), a further $3 \mathrm{~s}$ from the beginning of each trial was excluded to discard the initial easy phase of this task $(1, a, 2, b, 3, c$,$) . One power value for each$ condition was obtained by concatenating its data across trials and averaging across time points. For each pair of conditions (hard>easy, easy>rest, hard $>$ rest), the percentage signal change was computed as: [ (power in condition $1 /$ power in condition 2) - 1] $* 100$. 
For each electrode, a permutation testing approach was used to statistically compare power change across each pair of conditions. For each electrode, the instantaneous power timeseries of all task trials from both conditions were concatenated serially to form a loop in the same order in which they were conducted. To close the loop, the end of the last trial was joined to the beginning of the first trial. All trial onset/offset markers were then shifted using the same jitter (randomly generated for each permutation), allowing them to "rotate" along the data loop. This rotation approach was used to generate surrogate power data while preserving trial lengths and the temporal correlations in the data. After the rotation, we computed the mean power (for each condition) and power ratio (across conditions) based on the new trial markers. Applying this rotation approach on the timeseries of the power rather than the raw data ensured that there were no artefacts in the form of sudden power changes at the points of trials concatenation. This process was repeated 100,000 times to create a surrogate distribution against which two-tailed statistical significance could be calculated (percentile ranks 97.5 and 2.5) for each electrode.

To relate electrode locations with control-related regions identified by fMRI studies, a canonical resting-state fronto-parietal network (FPN) volumetric mask was used (Yeo et al., 2011). To match the original resolution of electrode localization using the grid method, the volumetric mask was resampled to $2 \times 2 \times 2$ $\mathrm{mm}$, then binarized to include any voxel with a non-zero probability. A volumetric approach was used rather than a surface-based approach because the presence of cortical lesions (i.e. the tumour) prevented accurate cortical surface extraction. 
Table 1. Patient demographics

\begin{tabular}{lllll}
\hline \multirow{2}{*}{ Patient } & Age & \multirow{2}{*}{ Sex } & \multicolumn{2}{c}{ Tumour } \\
\cline { 4 - 5 } & range & & hemisphere & Main location \\
\hline $\mathbf{1}$ & $21-30$ & Male & Left & Frontal \\
$\mathbf{3}$ & $21-30$ & Male & Left & Frontal \\
$\mathbf{4}$ & $41-50$ & Female & Left & Frontal \\
$\mathbf{5}$ & $21-30$ & Male & Left & Temporal \\
$\mathbf{6}$ & $51-60$ & Female & Left & Frontal \\
$\mathbf{7}$ & $21-30$ & Female & Right & Frontal \\
$\mathbf{8}$ & $21-30$ & Male & Right & Frontal \\
$\mathbf{9}$ & $41-50$ & Female & Right & Frontal \\
$\mathbf{1 0}$ & $21-30$ & Male & Left & Frontal \\
$\mathbf{1 1}$ & $31-40$ & Female & Left & Temporal \\
$\mathbf{1 2}$ & $21-30$ & Female & Left & Temporal \\
$\mathbf{1 3}$ & $51-60$ & Female & Left & Temporal \\
\hline & $21-30$ & Male & Left & Frontal \\
& & & & \\
\hline
\end{tabular}


medRxiv preprint doi: https://doi.org/10.1101/2021.08.13.21261980; this version posted August 13, 2021. The copyright holder for this preprint (which was not certified by peer review) is the author/funder, who has granted medRxiv a license to display the preprint in perpetuity.

\section{It is made available under a CC-BY 4.0 International license.}

a

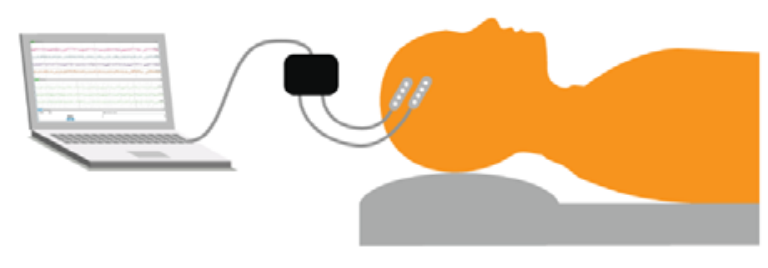

b

\author{
Baseline (rest) \\ No task, 2-3 mins \\ Simple counting (easy) \\ \begin{tabular}{l|l|l|lll}
1 & 2 & 3 & 4 & $\cdots$ & 20
\end{tabular} \\ Alternate counting (hard) \\ \begin{tabular}{l|l|l|l|l|}
1 & a & 2 & b & $\cdots$
\end{tabular}
}

c
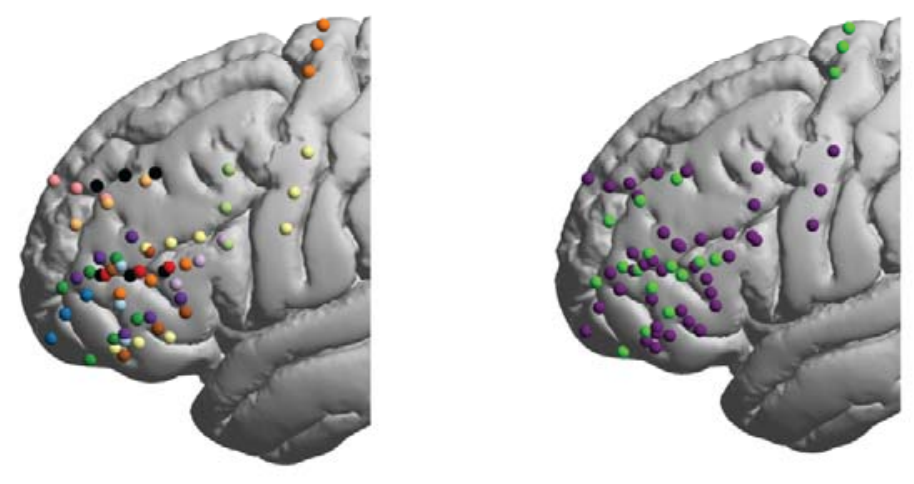

- Left hemisphere

- Right hemisphere

Figure 1. Intraoperative ECoG setup and electrode localization. (a) Intraoperative setup: Patient is awake during the three experimental conditions and the electrophysiological signals are simultaneously recorded using electrode strips placed directly on the cortical surface. (b) Experimental conditions: one rest (no task) and two verbal counting tasks. Easy task involved simple counting from 1 to 20. Hard task involved alternating between numbers and letters. (c) Left: Electrode distribution for each patient in a separate colour (13 patients, 59 electrodes after bipolar re-referencing). Right: hemispheric distribution of electrodes. 


\section{Results}

\section{Hard>easy contrast reveals local increases in gamma power contrasting with widespread decreases in beta power}

First we examined spectral power changes for the hard>easy contrast. As discussed above, this contrast commonly shows frontal control regions in fMRI studies. Based on previous reports in the literature, we focused on power changes in the two gamma bands (HG and LG) and the beta band. For the HG and LG bands, statistically significant electrodes showed predominantly $\mathrm{HG}$ and $\mathrm{LG}$ increases (Figure 2a, 2b) and almost all electrodes showing significant LG increases also showed HG increases $(92.3 \%$ ) (Figure 2a). In contrast, almost all significant changes in the beta band were power decreases (Figure a, 2b). Most electrodes showing significant $\mathrm{HG}$ and $\mathrm{LG}$ increases also showed significant beta decreases (85.1\% HG, $76.9 \%$ LG). While these results suggest that increases in gamma power co-occur with decreases in beta power, across all electrodes there were only modest correlations between gamma increases and beta decreases (for $\mathrm{HG}, \quad r=0.64$, $p<0.0001$; for LG, $r=0.32, p=0.005$ ).

Next we assessed the spatial distribution of these power modulations. Figure 2a, b shows circumscribed gamma power increases, while beta decreases are more broadly distributed across the recording area. To quantify this observation, we compared the Euclidean distances between all pairs of electrodes with significant power changes for each of the three bands (unpaired t-test, HG vs beta $\mathrm{t}_{877}=-7.8$, $p=1.5 \times 10^{-14}$, LG vs beta $\mathrm{t}_{604}=-2.2, p=0.03$; unpaired t-test after excluding electrodes lying on the motor cortex, HG vs beta $\mathrm{t}_{784}=-3.5, p=0.00046$, LG vs beta $t_{511}=-0.98$, $p=0.33$ ). Although this measure is, at least in part, affected by the spatial distribution of the electrodes, it nevertheless captures the distributed nature of the beta decreases compared to the more focal increases in the gamma range.

For completeness, we also examined power modulations in the lower frequency bands (delta, theta, alpha). These bands showed a similar picture to the beta band, with predominantly power decreases though with a patchier spatial arrangement (Supplementary Figure 1). Overall, these results show that the 
common finding of better spatial specificity for higher vs lower frequencies (Crone et al., 2006) extends into lateral prefrontal cortex.

To demonstrate that our selected canonical frequency bands do not bias the results, Figure 2c shows the average power spectral density (PSD), for each condition, of all the electrodes that showed significant changes in all three bands: HG and LG increases as well as beta decreases. Indeed, the power modulations computed using canonical frequency bands align closely with observed differences in PSDs between hard and easy tasks. Furthermore, the lack of PSD peaks in the gamma range suggests that the simultaneous power increases in HG and LG reflect a broadband process (Miller et al., 2009).

Taken together, these results show that, along the lateral frontal cortex, increased cognitive demand is associated with a localized increase in high frequency power and a spatially distributed decrease in low frequency power. 
a
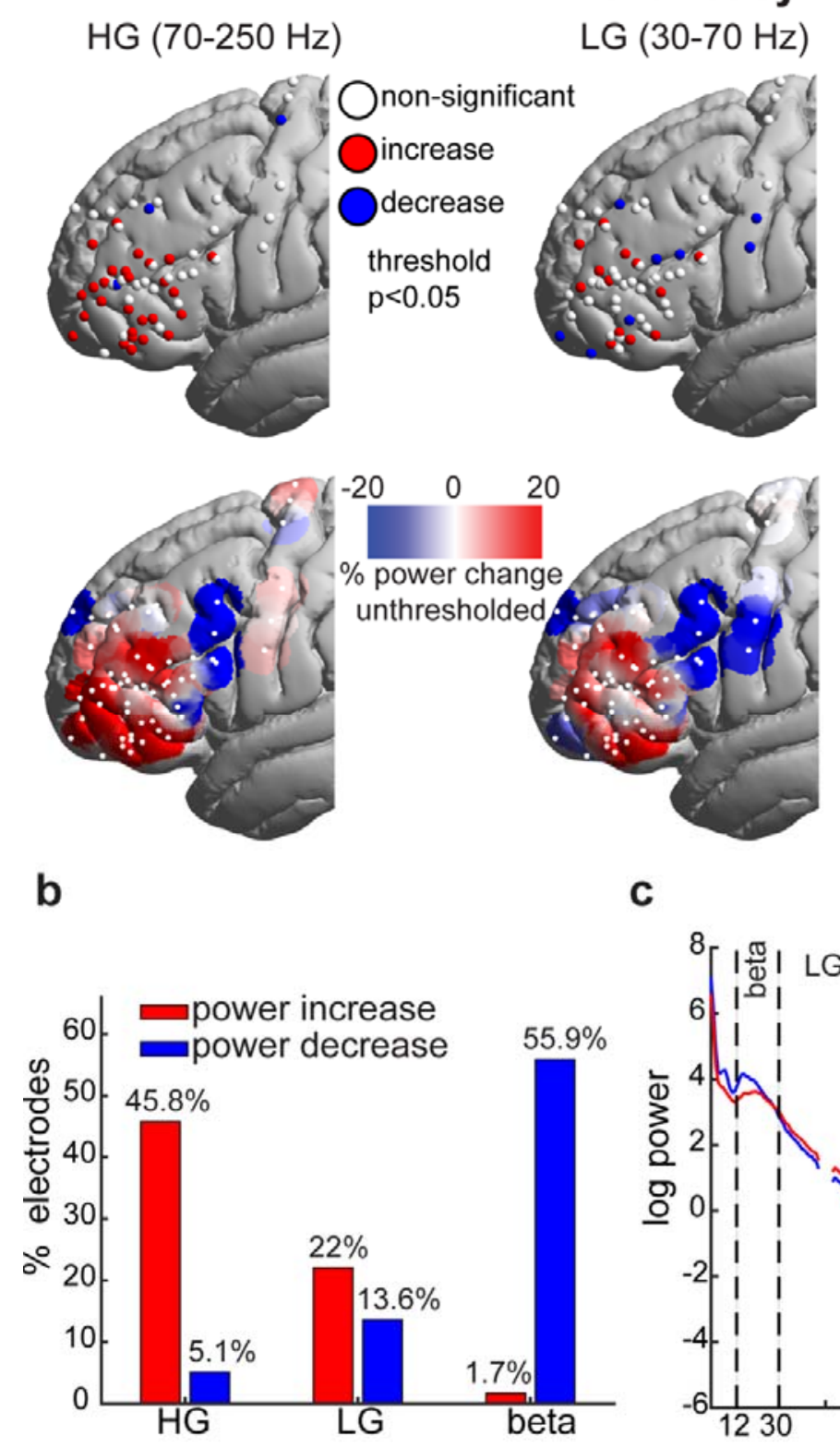

hard>easy
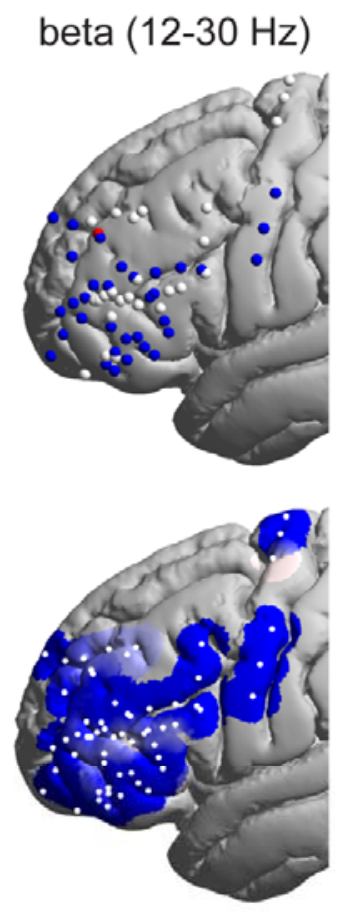

b

Figure 2. Hard>easy spectral power modulations. (a) Top: Electrodes with significant (thresholded at $p<0.05$, uncorrected) power increases (red), decreases (blue) and nonsignificant changes (white). Bottom: Unthresholded average smoothed data. Power for each electrode (white dots; including electrodes with non-significant power changes) was spatially smoothed by $10 \mathrm{~mm}$ and the value at each surface vertex is the average of the overlapping powers. (b) Proportion of electrodes (out of a total of 59) showing significant power modulations for each of the frequency bands. (c) Average of PSDs of all electrodes showing significant changes in all three bands: HG and LG power increases as well as beta power decreases. Notch filtered noise frequency bands (see methods) are hidden. 


\section{High frequency power increases overlap with the fMRI- defined fronto-parietal network}

The results so far highlight a localized group of electrodes with increases in gamma power surrounded by dorsal and posterior electrodes that show little to no power modulations (Figure 2a). This spatial pattern is reminiscent of fMRI studies identifying localized frontal control-related patches. Here we predicted that gamma increases in our data would overlap with a canonical mask of the control-related FPN as defined in fMRI data (taken from Yeo et al., 2011b; see Methods).

In our dataset, 33 electrodes were located within the FPN, while 26 lay outside this network (Figure 3a). Of the FPN electrodes, 61.3\% showed a significant $\mathrm{HG}$ increase in the hard>easy contrast, as compared to $28.6 \%$ of nonFPN electrodes (Figure 3b). LG increases showed a similar trend (29\% vs 14.3\%). In contrast, the proportions of electrodes showing beta power decreases were similar for FPN and non-FPN (51.6\% vs 60.7\%). Therefore, while power increases with increased demand were more likely within the FPN compared to outside the network, decreases in the beta band were equally likely both within and outside the network.

In a subsequent analysis, we examined data across all electrodes, irrespective of individual significance. Here too, $\mathrm{HG}$ and $\mathrm{LG}$ power increases were significantly stronger in FPN than in non-FPN electrodes (Figure 3c; unpaired t-test HG $\mathrm{t}_{57}=2.66, \quad p=0.005, \quad \mathrm{LG} \mathrm{t}_{57}=2.71, \quad p=0.004, \quad \mathrm{p}$-values are one-tailed due to our directional prediction of gamma increases within the FPN). Again, there was no significant difference between FPN and non-FPN electrodes for beta power decreases $\left(\mathrm{t}_{57}=1.2, p=0.2\right.$, two tailed as we had no specific directional prediction) nor for any of the lower frequency bands (two tailed $p>0.6$ ).

A limitation of the previous analysis is that most effects could be driven by a subset of patients. To address this, we used data from all the patients who each had electrodes overlapping with both FPN and non-FPN regions to perform a withinsubjects analysis (11 out of the 13 patients). Despite the lower number of patients available for this analysis, the results again showed that within the same patient, FPN electrodes showed statistically stronger LG power increases compared to nonFPN electrodes (Wilcoxon signed rank test; $p=0.02$, one tailed) and no differences 
(though statistically trending) for the beta power ( $p=0.08$, two tailed). Here, however, differences for $\mathrm{HG}$ were not significant $(p=0.2$, one tailed). These results hold even after excluding the apparent outlier patient in Figure 3d (HG $p=0.19$; LG $p=0.01$; both one tailed; beta $p=0.049$ two tailed).

Collectively, these results suggest that demand-related power increases in high frequency bands co-localize with the fMRI-identified FPN. Thus, results from two different modalities (ECoG and fMRI) converge in defining a localized region within the lateral frontal lobe related to cognitive control. 
a

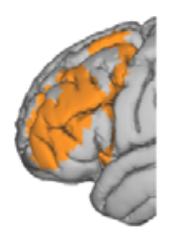

C
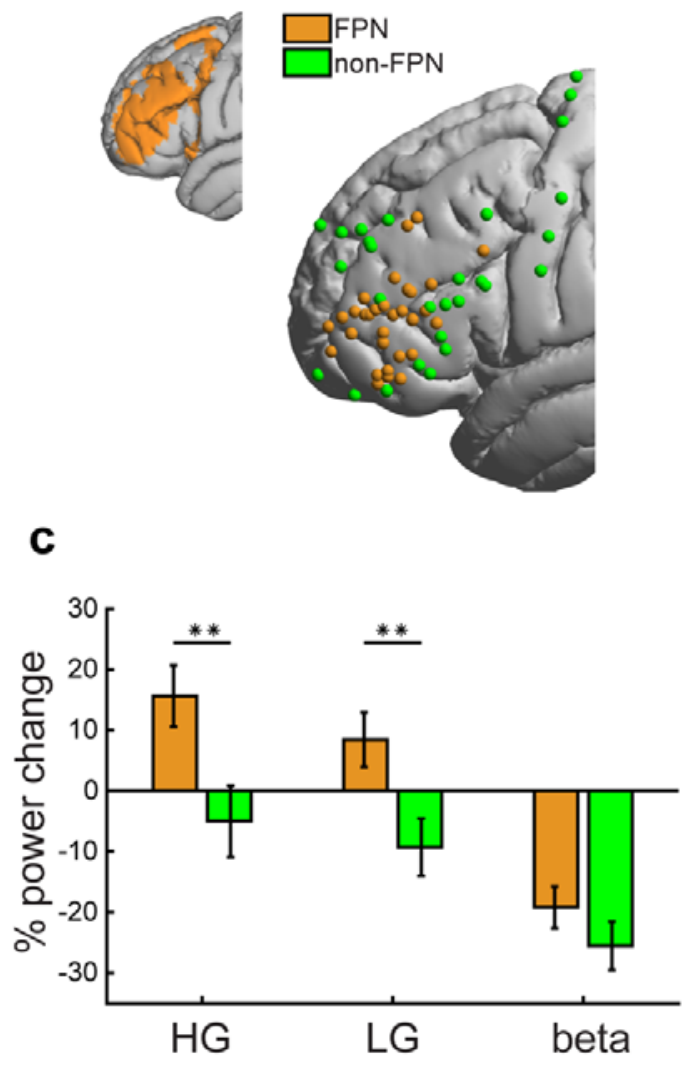

b

d

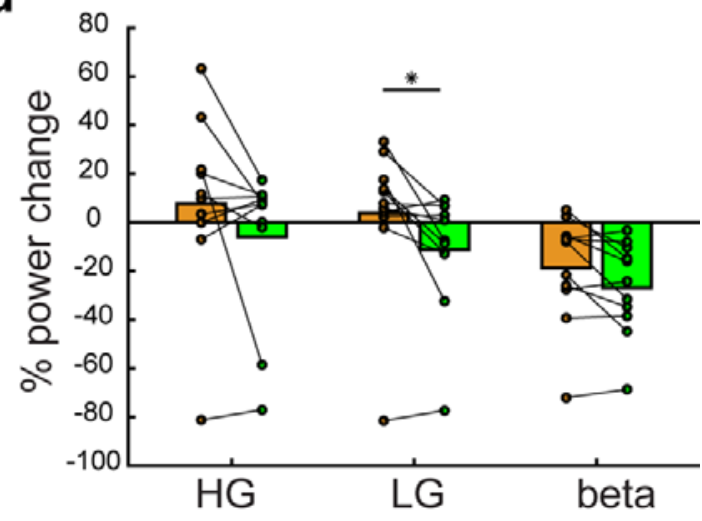

Figure 3. Broadband gamma power increases overlap with FPN. (a) Electrodes coloured based on their overlap with the canonical fMRI FPN mask in volume space. Top left inset: A cortical surface visualization of the volumetric canonical fMRI FPN mask (Yeo et al., 2011) (Note: this is for illustrative purposes only and may include slight distortions resulting from volume-to-surface transformation). (b) Percentage of electrodes showing significant power modulations out of all electrodes located within (orange and yellow) and outside (dark and light green) the FPN. Darker coloured bars (above the zero line) show percentage of electrodes showing power increases. Lighter coloured bars (below the zero line) show percentage of electrodes showing power decreases. (c) Power modulations of all electrodes within and outside the FPN for each frequency band. $*_{*} p<0.01$, one tailed, unpaired t-test. (d) A within-subjects comparison of power changes between FPN and non-FPN electrodes. Each dot represents average power across each group of electrodes for one patient. ${ }^{*} p<0.05$, for one tailed tests only, Wilcoxon signed rank test. 


\section{Power modulations in the easy>rest contrast}

Next we sought to assess whether the localized gamma increases, identified in the hard>easy contrast where the conditions are matched on multiple aspects (e.g. speech, counting), would also replicate in the easy>rest contrast where those aspects are not matched.

The distribution of $\mathrm{HG}, \mathrm{LG}$ and beta changes in the easy>rest contrast is shown in Figure 4a. Across all electrodes, there was no significant correlation between gamma power increases for hard>easy and easy>rest (HG: $r=-0.07 p=0.53$; LG: $r=-0.09 p=0.42$ ), indicating different topographical arrangement for the gamma modulations in the two contrasts. For beta power decreases there was a weak correlation $(r=0.22, p=0.045)$. In contrast to the results for the hard>easy contrast, the percentage of electrodes with significant gamma increases was similar between FPN and non-FPN electrodes (Figure 4b, HG 32.3\% vs 39.3\%; LG $22.6 \%$ vs 17.9\%). Similarly, neither the average power modulations across electrodes (Figure 4c; unpaired t-test $\mathrm{HG} \mathrm{t}_{57}=-1.28, \quad p=0.90, \quad$ LG $\mathrm{t}_{57}=-0.67, p=0.78$, one-tailed; beta $\mathrm{t}_{57}=-0.69, p=0.49$ two tailed) nor the within-subject analysis (Figure 4d; Wilcoxon signed rank test, HG $p=0.86, L G p=0.58$, one tailed for both; beta $p=0.52$ two tailed,) showed any significant differences between FPN and non-FPN electrodes across the three bands. Again these results were stable after excluding the apparent outlier patient in Figure 4d (within-subject analysis: Wilcoxon signed rank test, HG $p=0.99$; $L G p=0.75$, one tailed for both; beta $p=0.77$ two tailed).

Interestingly, easy>rest gamma increases were shifted posteriorly to hard>easy increases (Figure 4e). To quantify this, we compared y-coordinates (anterior-posterior axis) of electrodes showing significant gamma increases between hard>easy and easy>rest contrasts. The results confirmed a posterior shift for the group of significant $\mathrm{HG}$ electrodes in the easy>rest contrast (Wilcoxon signed rank test, HG $p=0.02$, LG $p=0.07$ ).

Altogether, these results confirm that the hard>easy contrast is most suitable for revealing localized control-related gamma frontal regions. 


\section{easy>rest}

a HG

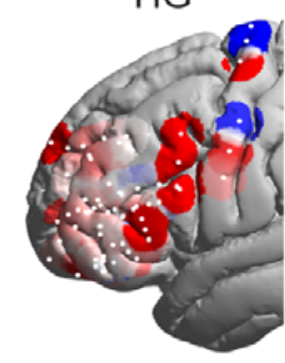

b

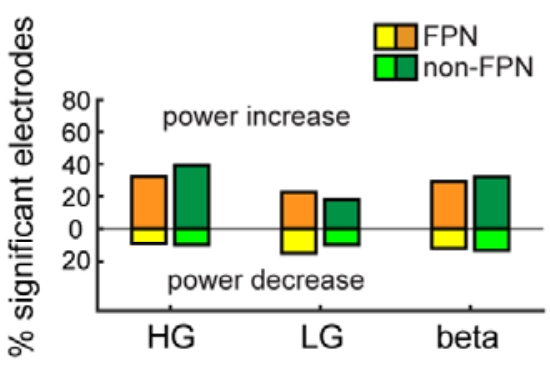

d

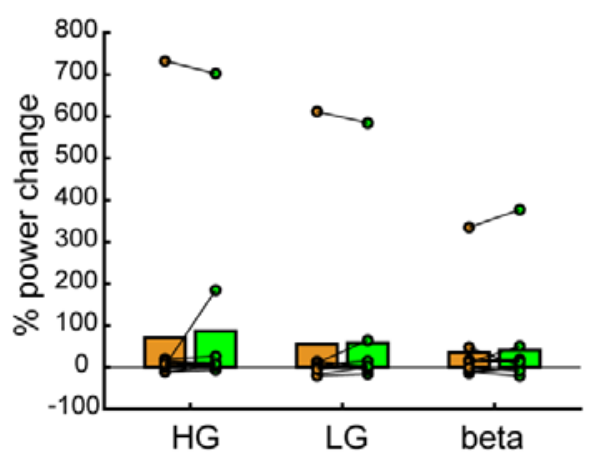

LG

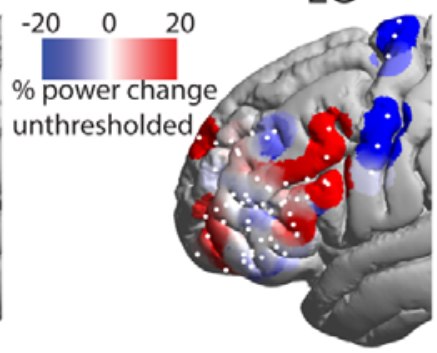

beta

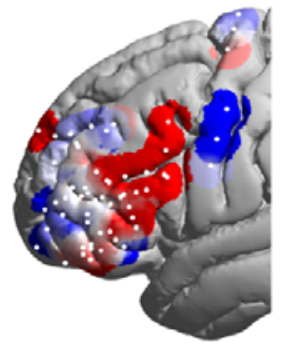

C

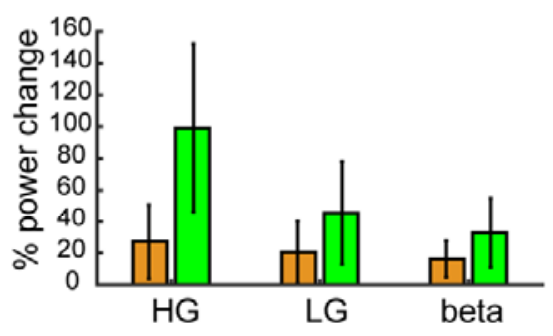

e

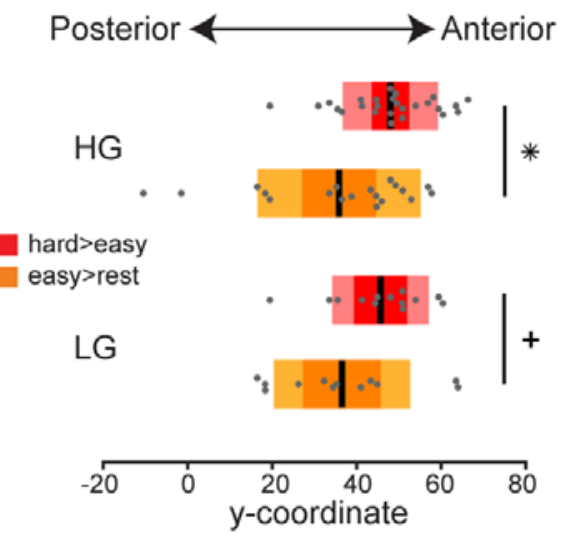

Figure 4. Power modulations for the easy>rest contrast. (a) Surface projection of power modulations for the easy>rest contrast. Remaining details are identical to Figure 2a. (b-d) Comparison of easy>rest power changes between FPN and non-FPN electrodes. Details are identical to Figure 3(b-d). (e) Box plots of y-coordinate of electrodes with significant power increases. Middle black line: mean; darker box limits: 1 SD; lighter box limits: $95 \%$ CI. ${ }^{*} p<0.05+p=0.07$, using Wilcoxon rank sum test. 


\section{Discussion}

We recorded LFP signals using ECoG from the lateral frontal surface of human patients undergoing awake craniotomies for tumour resections. The results revealed a circumscribed frontal region that shows increases in broadband gamma power with increased task demands. Localised gamma increases were accompanied by spatially broad beta power decreases. Electrodes showing gamma increases overlapped with a canonical mask of the fMRI-defined FPN. Our results confirm that specific control regions of the lateral frontal surface, previously defined by fMRI, can also be identified using clinically-relevant electrophysiological recording.

\section{Delineating lateral frontal control regions using converging evidence from ECoG and fMRI}

There is some evidence from previous electrophysiology studies that gamma power increases in frontal regions are associated with multiple executive processes such as attention, working memory and abstract rules (Fries, 2001; Ray et al., 2008; Szczepanski et al., 2014; Helfrich and Knight, 2016). However, invasive human studies have rarely systematically assessed the anatomical distribution of gamma increases, with most studies reporting isolated findings in individual electrodes (Lachaux et al., 2012; Helfrich and Knight, 2016). This lack of detailed topographical assessment limits our ability to relate findings across studies and across neuroimaging modalities. Here we demonstrate that a circumscribed region along the lateral frontal surface robustly shows increases in gamma power during an executive task. Although some previous studies have reported different reliabilities and putative physiological origins for $\mathrm{HG}$ and LG (Crone et al., 2006; Ray and Maunsell, 2011; Buzsáki and Wang, 2012; Lachaux et al., 2012), in our data both gamma bands were broadly in agreement.

Our results suggest converging evidence from ECoG and fMRI modalities for control-related parcellation of the lateral frontal cortex. We used a task demand manipulation similar to the one that was previously shown to recruit control regions in fMRI studies (Fedorenko et al., 2013; Assem et al., 2020; Shashidhara et al., 
2020) and the observed gamma power increases associated with increased demand co-localized with a canonical FPN derived from independent resting-state fMRI data from healthy participants. Previous studies have linked localized gamma increases with corresponding fMRI activations in early cortical regions (Nir et al., 2007; Engell et al., 2012; Hermes et al., 2012a). Accordingly, gamma increases were generally interpreted to reflect localized task-related processing. The current results extend these reports to the domain of cognitive control in frontal regions.

In contrast to focal increases in the gamma range, we observed spatially broad decreases in power in the beta band (and other lower frequencies) which were not confined to the FPN. It has been previously shown that synchronization in lower frequency bands between fronto-parietal regions improved during executive tasks e.g. (Voytek et al., 2015). Our findings suggest that increases in beta synchrony may be accompanied by decreases in power. Concerning the relation between gamma increases and beta decreases, one suggestion is that these reflect two sides of the same process, a rotated power spectrum around a middle range frequency (Podvalny et al., 2015; Helfrich and Knight, 2016). Recent evidence, however, argues against this simple interpretation, showing that depending on the cortical region, increases in high gamma power are not necessarily accompanied by decreases in low frequency power (Fellner et al., 2019). In line with this, the current results also showed that beta decreases were more spatially broad and were not necessarily accompanied by gamma increases. Another framework proposes a hybrid spiking-synaptic plasticity working memory model, in which bursts of spikes (gamma increases) in superficial layers serve to encode and maintain working memory content, while beta, which is assumed to have an inhibitory role, is suppressed in deeper layers to allow superficial gamma bursts (Lundqvist et al., 2011; Miller et al., 2018). This model is specific for regions that are involved in working memory processes, and our results are in line with this suggestion within the control-related areas. As it stands, however, the model does not predict decreased beta power in regions outside the FPN. Pending further experimental and theoretical studies, our results provide an important constraint for physiologicallymotivated models of executive processes.

Power modulations in the gamma range for the easy>rest contrast revealed posterior shift of gamma increases compared to the hard>easy contrast. One possibility is that this shift mirrors fMRI studies showing an anterior-posterior 
medRxiv preprint doi: https://doi.org/10.1101/2021.08.13.21261980; this version posted August 13, 2021. The copyright holder for this preprint

cognitive demand gradient across the lateral frontal surface, with easier tasks involving more posterior regions and more challenging tasks recruiting more anterior regions (Badre and Nee, 2018; Shashidhara et al., 2019; Assem et al., 2020). Another possibility is related to the conditions that comprise the two contrasts. While the hard>easy contrast compared conditions that are closely matched on features such as speech and counting task, the easy>rest contrast is less controlled. Accordingly, changes in activity may be related to several differences between the conditions, most prominently speech production (e.g., the easy condition includes speech while the patient remains silent during the rest condition). Plausibly, posterior gamma increases might also reflect articulation or language related processing in the posterior inferior frontal gyrus (Basilakos et al., 2018).

\section{Clinical potential}

Our findings open the door for extending clinical functional mapping to the domain of cognitive/executive control during tumour resection surgeries. Damage to control regions is associated with disorganized behaviour (Glascher et al., 2010, 2012, Woolgar et al., 2010, 2018; Warren et al., 2014) and poorer recovery following neurosurgery (Romero-Garcia et al., 2019). During surgeries, electrical stimulation is commonly used to map motor and language functions, where activity is confined to well-defined areas with clear behavioural responses, so that resection of eloquent tissue can be avoided. In higher association areas and in particular for executive control regions, the use of stimulation is more challenging, primarily because of the complex nature of the mapped functions and the distributed areas that support them. In addition, intraoperative stimulation is time-consuming while neurosurgeons search for the areas responsive to the tested functions with increased risk of seizures. Current mapping approaches to assess executive regions intraoperatively are limited, with only sparse evidence where direct electrical stimulation was used (Wager et al., 2013; Puglisi et al., 2018, 2019). ECoG has the potential to provide complementary information to guide stimulation and clinical decision making, especially for executive control functions. Most ECoG studies that investigate cognitive control are conducted at the patient's bedside between two surgeries as part of epilepsy treatment, and usually use complex computer-based tasks that are not suitable for an intraoperative setting. Here we employed a simple behavioural 
manipulation based on task demand which was well tolerated by most patients and the whole testing was performed in under 10 minutes. The link of increased gamma power to the FPN establishes a critical anatomo-functional foundation for this approach. These results also suggest the FPN remains relatively intact next to tumour tissue. Further, the detected gamma signals are characterized by spatial specificity of a few millimetres. For example, nearby electrodes on the same strip can show differential gamma responses. This level of neuroanatomical precision is vital for guiding neurosurgeons during tumour excision.

Exploring several future avenues will help scale up this approach for clinical mapping. For example, while the current study aimed to detect an index of cognitive control processing, only one type of cognitive demand was manipulated. A conjunction of activity across several tasks is more likely to focus on core domaingeneral control regions (Assem et al., 2020). This will require modifying existing paradigms to more surgical theatre friendly versions. One such task could be contrasting verb generation with repeating nouns. Relatedly, our electrodes coverage was mostly limited to the middle and inferior frontal gyri. It will be clinically useful to extend the current findings to other components of the FPN (frontal pole, dorsal frontal and lateral parietal), for example in cases of different or distributed tumour locations. Similarly, a denser and more distributed set of electrodes would allow investigating electrophysiological signals in fMRI-based networks neighbouring the FPN. Further, only amplitude modulations were investigated in this study. Investigating synchrony within and across frequency bands (e.g., phase changes, phase-amplitude coupling) could reveal unique markers of FPN dynamics during task execution. Precise neurosurgical resection would also benefit from individually specific fMRI defined FPN (Shashidhara et al., 2020). This is complicated by our poor understanding of how brain tumours affect BOLD signal and shift the topographies of functional networks. Ultimately, integrating ECoG with brain stimulation and pre-operative multi-modal brain imaging opens up a new exciting phase for comprehensive surgical planning (Hart et al., 2020). 


\section{Acknowledgments}

The Royal Society provided financial support in the form of a Royal Society Dorothy Hodgkin Research Fellowship to YE (DH130100). Cambridge Commonwealth European and International Trust provided financial support in the form of a Yousef Jameel scholarship to MA. Guarantors of Brain provided financial support in the form of a Post-Doctoral Fellowship award to RRG. The National Institute for Health Research (NIHR, UK) provided financial support in the form of a Clinician Scientist Award 35 to SJP (ref: NIHR/CS/009/011). The Brain Tumour Charity provided financial support in the form of a grant award to MGH (ref: RG86218). J.D was funded by a Medical Research Council grant (SUAG/045.G101400) All the sponsors had no role in the design or conduct of this research. We thank Mallory Owen for help with administering and organising neuropsychological tests from the patients.

\section{Data Availability}

Due to clinical ethical considerations and in accordance with the ethics approval for the study by the East of England - Cambridge Central Research Ethics Committee (REC reference 16/EE/0151), the data cannot be shared or become publicly available. 
medRxiv preprint doi: https://doi.org/10.1101/2021.08.13.21261980; this version posted August 13, 2021. The copyright holder for this preprint (which was not certified by peer review) is the author/funder, who has granted medRxiv a license to display the preprint in perpetuity.

It is made available under a CC-BY 4.0 International license .

\section{Supplementary Figures}
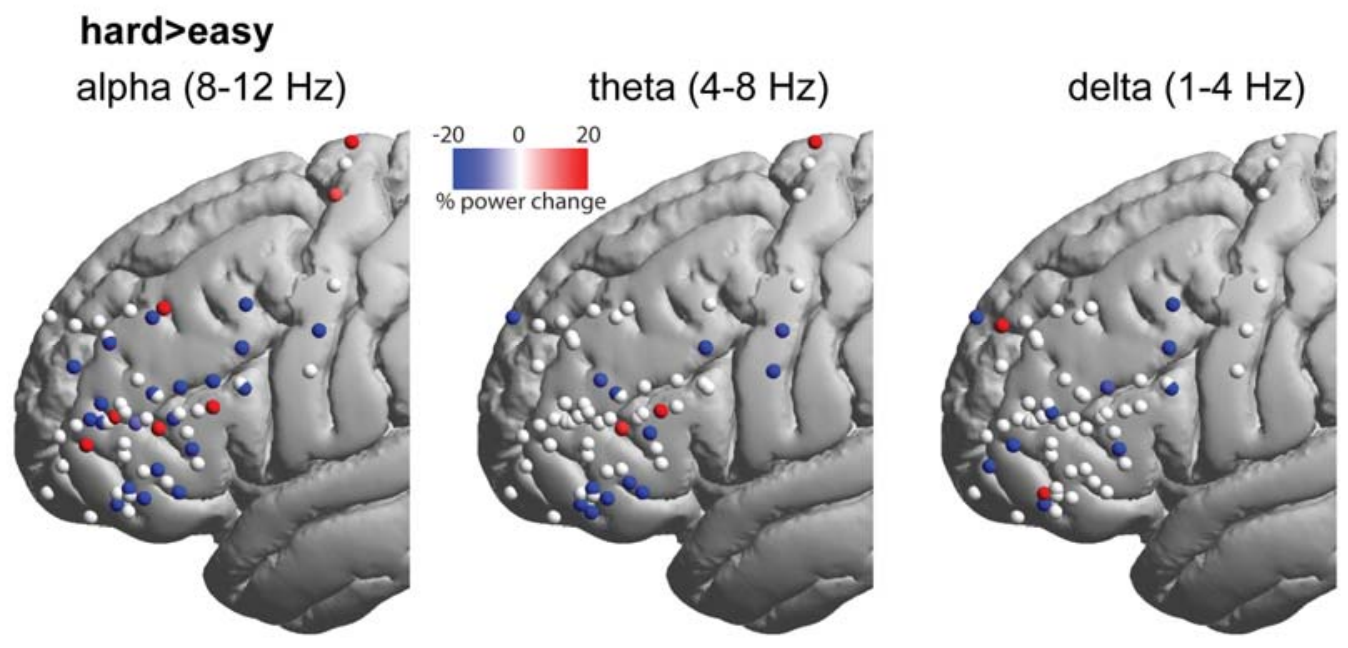

Supplementary Figure 1. Hard>easy power modulations in lower frequency bands. Electrodes with significant $(p<0.05$, uncorrected) power increases (red), decreases (blue) and non-significant changes (white). 


\section{References}

Assem M, Glasser MF, Van Essen DC, Duncan J. A Domain-General Cognitive Core Defined in Multimodally Parcellated Human Cortex. Cereb Cortex 2020; 30: 4361-80.

Badre D, Nee DE. Frontal Cortex and the Hierarchical Control of Behavior. Trends Cogn Sci 2018; 22: 170-88.

Basilakos A, Smith KG, Fillmore P, Fridriksson J, Fedorenko E. Functional Characterization of the Human Speech Articulation Network. Cereb Cortex 2018; 28: 1816-30.

Botvinik-Nezer R, Holzmeister F, Camerer CF, Dreber A, Huber J, Johannesson M, et al. Variability in the analysis of a single neuroimaging dataset by many teams. Nature 2020; 582: 84-8.

Bouyer J., Montaron M., Rougeul A. Fast fronto-parietal rhythms during combined focused attentive behaviour and immobility in cat: cortical and thalamic localizations. Electroencephalogr Clin Neurophysiol 1981; 51: 244-52.

Buzsáki G, Wang X-J. Mechanisms of Gamma Oscillations. Annu Rev Neurosci 2012; 35: 203-25.

Coalson TS, Essen DC Van, Glasser MF. The impact of traditional neuroimaging methods on the spatial localization of cortical areas. Proc Natl Acad Sci 2018; 115: E6356-65.

Cole MW, Ito T, Braver TS. The Behavioral Relevance of Task Information in Human Prefrontal Cortex. Cereb Cortex 2016; 26: 2497-505.

Cole MW, Schneider W. The cognitive control network: Integrated cortical regions with dissociable functions. Neuroimage 2007; 37: 343-60.

Corbetta M, Shulman GL. Control of goal-directed and stimulus-driven attention in the brain. Nat Rev Neurosci 2002; 3: 201-15.

Crone NE, Sinai A, Korzeniewska A. High-frequency gamma oscillations and human brain mapping with electrocorticography. In: Progress in Brain Research. 2006. p. 275-95

Diamond A. Executive Functions. Annu Rev Psychol 2013; 64: 135-68.

Duncan J. The multiple-demand (MD) system of the primate brain: mental programs for intelligent behaviour. Trends Cogn Sci 2010; 14: 172-9.

Duncan J, Owen AM. Common regions of the human frontal lobe recruited by diverse cognitive demands. Trends Neurosci 2000; 23: 475-83.

Engell AD, Huettel S, McCarthy G. The fMRI BOLD signal tracks electrophysiological spectral perturbations, not event-related potentials. Neuroimage 2012; 59: 2600-6.

Erez Y, Assem M, Coelho P, Romero-Garcia R, Owen M, McDonald A, et al. Intraoperative mapping of executive function using electrocorticography for patients with 
low-grade gliomas. Acta Neurochir (Wien) 2021; 163: 1299-309.

Erez Y, Duncan J. Discrimination of Visual Categories Based on Behavioral Relevance in Widespread Regions of Frontoparietal Cortex. J Neurosci 2015; 35: 12383-93.

Fedorenko E, Duncan J, Kanwisher N. Broad domain generality in focal regions of frontal and parietal cortex. Proc Natl Acad Sci 2013; 110: 16616-21.

Fellner M-C, Gollwitzer S, Rampp S, Kreiselmeyr G, Bush D, Diehl B, et al. Spectral fingerprints or spectral tilt? Evidence for distinct oscillatory signatures of memory formation. PLOS Biol 2019; 17: e3000403.

Fries P. Modulation of Oscillatory Neuronal Synchronization by Selective Visual Attention. Science (80- ) 2001; 291: 1560-3.

Glascher J, Adolphs R, Damasio H, Bechara A, Rudrauf D, Calamia M, et al. Lesion mapping of cognitive control and value-based decision making in the prefrontal cortex. Proc Natl Acad Sci 2012; 109: 14681-6.

Glascher J, Rudrauf D, Colom R, Paul LK, Tranel D, Damasio H, et al. Distributed neural system for general intelligence revealed by lesion mapping. Proc Natl Acad Sci 2010; 107: 4705-9.

Glasser MF, Coalson TS, Robinson EC, Hacker CD, Harwell J, Yacoub E, et al. A multimodal parcellation of human cerebral cortex. Nature 2016; 536: 171-8.

Haller M, Case J, Crone NE, Chang EF, King-Stephens D, Laxer KD, et al. Persistent neuronal activity in human prefrontal cortex links perception and action. Nat Hum Behav 2018; 2: 80-91.

Hart MG, Romero-Garcia R, Price SJ, Santarius T, Suckling J. Connections, Tracts, Fractals, and the Rest: A Working Guide to Network and Connectivity Studies in Neurosurgery. World Neurosurg 2020

Havas V, Gabarrós A, Rifa-ros X, Plans G. Brain \& Language Electrical stimulation mapping of nouns and verbs in Broca' $s$ area. 2015; 146: 53-63.

Helfrich RF, Knight RT. Oscillatory Dynamics of Prefrontal Cognitive Control. Trends Cogn Sci 2016; 20: 916-30.

Hermes D, Miller KJ, Noordmans HJ, Vansteensel MJ, Ramsey NF. Automated electrocorticographic electrode localization on individually rendered brain surfaces. $\mathbf{J}$ Neurosci Methods 2010; 185: 293-8.

Hermes D, Miller KJ, Vansteensel MJ, Aarnoutse EJ, Leijten FSS, Ramsey NF. Neurophysiologic correlates of fMRI in human motor cortex. Hum Brain Mapp 2012; 33: 1689-99.

Hermes D, Siero JCW, Aarnoutse EJ, Leijten FSS, Petridou N, Ramsey NF. Dissociation between Neuronal Activity in Sensorimotor Cortex and Hand Movement Revealed as a Function of Movement Rate. J Neurosci 2012; 32: 9736-44. 
medRxiv preprint doi: https://doi.org/10.1101/2021.08.13.21261980; this version posted August 13, 2021. The copyright holder for this preprint (which was not certified by peer review) is the author/funder, who has granted medRxiv a license to display the preprint in perpetuity.

It is made available under a CC-BY 4.0 International license .

Howard MW. Gamma Oscillations Correlate with Working Memory Load in Humans. Cereb Cortex 2003; 13: 1369-74.

Jackson J, Rich AN, Williams MA, Woolgar A. Feature-selective Attention in Frontoparietal Cortex: Multivoxel Codes Adjust to Prioritize Task-relevant Information. J Cogn Neurosci 2016; 29: 310-21.

Jenkinson M, Beckmann CF, Behrens TEJ, Woolrich MW, Smith SM. FSL. Neuroimage 2012; 62: 782-90.

Lachaux J-P, Axmacher N, Mormann F, Halgren E, Crone NE. High-frequency neural activity and human cognition: Past, present and possible future of intracranial EEG research. Prog Neurobiol 2012; 98: 279-301.

Logothetis NK, Pauls J, Augath M, Trinath T, Oeltermann A. Neurophysiological investigation of the basis of the fMRI signal. Nature 2001; 412: 150-7.

van Loon EMP, Heijenbrok-Kal MH, van Loon WS, van den Bent MJ, Vincent AJPE, de Koning I, et al. Assessment methods and prevalence of cognitive dysfunction in patients with low-grade glioma: A systematic review. J Rehabil Med 2015; 47: 481-8.

Lundqvist M, Herman P, Lansner A. Theta and Gamma Power Increases and Alpha/Beta Power Decreases with Memory Load in an Attractor Network Model. J Cogn Neurosci 2011; 23: 3008-20.

Lundqvist M, Rose J, Herman P, Brincat SLL, Buschman TJJ, Miller EKK. Gamma and Beta Bursts Underlie Working Memory. Neuron 2016; 90: 152-64.

Mainy N, Kahane P, Minotti L, Hoffmann D, Bertrand O, Lachaux J-P. Neural correlates of consolidation in working memory. Hum Brain Mapp 2007; 28: 183-93.

Miller EK, Lundqvist M, Bastos AM. Working Memory 2.0. Neuron 2018; 100: 463-75.

Miller KJ, Sorensen LB, Ojemann JG, den Nijs M. Power-Law Scaling in the Brain Surface Electric Potential. PLoS Comput Biol 2009; 5: e1000609.

Muhle-Karbe PS, De Baene W, Brass M. Do tasks matter in task switching? Dissociating domain-general from context-specific brain activity. Neuroimage 2014; 99: 332-41.

Mukamel R, Gelbard H, Arieli A, Hasson U, Fried I, Malach R. Coupling Between Neuronal Firing, Field Potentials, and fMRI in Human Auditory Cortex. Science (80- ) 2005; 309: 951-4.

Nir Y, Fisch L, Mukamel R, Gelbard-Sagiv H, Arieli A, Fried I, et al. Coupling between Neuronal Firing Rate, Gamma LFP, and BOLD fMRI Is Related to Interneuronal Correlations. Curr Biol 2007; 17: 1275-85.

Ojemann G, Ojemann J, Lettich E, Berger M. Cortical language localization in left, dominant hemisphere. J Neurosurg 1989; 71: 316-26.

Podvalny E, Noy N, Harel M, Bickel S, Chechik G, Schroeder CE, et al. A unifying principle underlying the extracellular field potential spectral responses in the human cortex. 
J Neurophysiol 2015; 114: 505-19.

Power JD, Cohen AL, Nelson SM, Wig GS, Barnes KA, Church JA, et al. Functional Network Organization of the Human Brain. Neuron 2011; 72: 665-78.

Puglisi G, Howells H, Sciortino T, Leonetti A, Rossi M, Conti Nibali M, et al. Frontal pathways in cognitive control: direct evidence from intraoperative stimulation and diffusion tractography. Brain 2019; 142: 2451-65.

Puglisi G, Sciortino T, Rossi M, Leonetti A, Fornia L, Conti Nibali M, et al. Preserving executive functions in nondominant frontal lobe glioma surgery: an intraoperative tool. $\mathbf{J}$ Neurosurg 2018; 131: 474-80.

Ray S, Maunsell JHR. Different Origins of Gamma Rhythm and High-Gamma Activity in Macaque Visual Cortex. PLoS Biol 2011; 9: e1000610.

Ray S, Niebur E, Hsiao SS, Sinai A, Crone NE. High-frequency gamma activity (80$150 \mathrm{~Hz}$ ) is increased in human cortex during selective attention. Clin Neurophysiol 2008; 119: 116-33.

Romero-Garcia R, Suckling J, Owen M, Assem M, Sinha R, Coelho P, et al. Disruptive and protective outcomes to memory and attention when treating diffuse glioma. medRxiv 2019: 19008581.

Shashidhara S, Mitchell DJ, Erez Y, Duncan J. Progressive Recruitment of the Frontoparietal Multiple-demand System with Increased Task Complexity, Time Pressure, and Reward. J Cogn Neurosci 2019; 31: 1617-30.

Shashidhara S, Spronkers FS, Erez Y. Individual-subject Functional Localization Increases Univariate Activation but Not Multivariate Pattern Discriminability in the 'Multipledemand' Frontoparietal Network. J Cogn Neurosci 2020; 32: 1348-68.

Siero JC, Hermes D, Hoogduin H, Luijten PR, Petridou N, Ramsey NF. BOLD consistently matches electrophysiology in human sensorimotor cortex at increasing movement rates: a combined 7T fMRI and ECoG study on neurovascular coupling. J Cereb Blood Flow Metab 2013; 33: 1448-56.

Stolk A, Griffin S, van der Meij R, Dewar C, Saez I, Lin JJ, et al. Integrated analysis of anatomical and electrophysiological human intracranial data. Nat Protoc 2018; 13: 1699723.

Szczepanski SM, Crone NE, Kuperman RA, Auguste KI, Parvizi J, Knight RT. Dynamic Changes in Phase-Amplitude Coupling Facilitate Spatial Attention Control in FrontoParietal Cortex. PLoS Biol 2014; 12: e1001936.

Voytek B, Kayser AS, Badre D, Fegen D, Chang EF, Crone NE, et al. Oscillatory dynamics coordinating human frontal networks in support of goal maintenance. Nat Neurosci 2015; 18: $1318-24$.

Wager M, Du Boisgueheneuc F, Pluchon C, Bouyer C, Stal V, Bataille B, et al. 
Intraoperative Monitoring of an Aspect of Executive Functions. Oper Neurosurg 2013; 72: $169-81$.

Warren DE, Power JD, Bruss J, Denburg NL, Waldron EJ, Sun H, et al. Network measures predict neuropsychological outcome after brain injury. Proc Natl Acad Sci 2014; 111: $14247-52$.

Woolgar A, Duncan J, Manes F, Fedorenko E. Fluid intelligence is supported by the multiple-demand system not the language system. Nat Hum Behav 2018; 2: 200-4.

Woolgar A, Parr A, Cusack R, Thompson R, Nimmo-Smith I, Torralva T, et al. Fluid intelligence loss linked to restricted regions of damage within frontal and parietal cortex. Proc Natl Acad Sci U S A 2010; 107: 14899-902.

Yeo BTT, Krienen FM, Sepulcre J, Sabuncu MR, Lashkari D, Hollinshead M, et al. The organization of the human cerebral cortex estimated by intrinsic functional connectivity. J Neurophysiol 2011; 106: 1125-65. 